\title{
Influence of World and Gravity Model Selection on Surface Interacting Vehicle Simulations
}

\author{
Michael M. Madden ${ }^{*}$ \\ NASA, Hampton, VA, 23681
}

\begin{abstract}
A vehicle simulation is "surface-interacting" if the state of the vehicle (position, velocity, and acceleration) relative to the surface is important. Surface-interacting simulations perform ascent, entry, descent, landing, surface travel, or atmospheric flight. Modeling of gravity is an influential environmental factor for surface-interacting simulations. Gravity is the free-fall acceleration observed from a world-fixed frame that rotates with the world. Thus, gravity is the sum of gravitation and the centrifugal acceleration due to the world's rotation. In surface-interacting simulations, the fidelity of gravity at heights above the surface is more significant than gravity fidelity at locations in inertial space. A surface-interacting simulation cannot treat the gravity model separately from the world model, which simulates the motion and shape of the world. The world model's simulation of the world's rotation, or lack thereof, produces the centrifugal acceleration component of gravity. The world model's reproduction of the world's shape will produce different positions relative to the world center for a given height above the surface. These differences produce variations in the gravitation component of gravity. This paper examines the actual performance of world and gravity/gravitation pairs in a simulation using the Earth.
\end{abstract}

\section{Nomenclature and Acronyms}

$\vec{\gamma}=$ the gravitational acceleration vector

$\omega=$ rotation of the world about its $\mathrm{z}$ axis

$\phi \quad=$ geodetic latitude

$\phi_{\mathrm{g}} \quad=$ geocentric latitude

a $\quad=$ semi-major axis of the world's reference ellipse

$\mathrm{b}=$ semi-minor axis of the world's reference ellipse

$\mathrm{C}_{\mathrm{n}, \mathrm{m}}=$ spherical harmonic coefficient (degree $\mathrm{n}$, order $\mathrm{m}$ ) of the gravitational potential

$\mathrm{C}_{\mathrm{n}} \quad=$ zonal spherical harmonic of the gravitational potential (equal to $\mathrm{C}_{\mathrm{n}, 0}$ )

e $\quad=$ eccentricity of the world's reference ellipse

$\mathrm{E} / \mathrm{C}=$ Rotating ellipsoid Earth model with constant gravitation

$\mathrm{E} / \mathrm{PM}=$ Rotating ellipsoid Earth model with point-mass gravitation

$\mathrm{E} / \mathrm{SE}=$ Rotating ellipsoid Earth model with symmetric ellipsoid gravitation

$\mathrm{ENR} / \mathrm{C}=$ Non-rotating ellipsoid Earth model with constant gravity

$\mathrm{ENR} / \mathrm{FA}=$ Non-rotating ellipsoid Earth model with free-air reduction gravity

$\mathrm{f}=$ flattening of the world surface

$\mathrm{ft} \quad=$ feet

$\mathrm{G} \quad=$ universal gravitation constant

$\mathrm{g}^{\mathrm{s}} \quad=$ acceleration due to gravity on the surface

$\mathrm{h}=$ height of an object above the surface

$\mathrm{I}_{\mathrm{xx}}, \mathrm{I}_{\mathrm{zz}}=$ moments of inertia for the world

$\mathrm{J}_{2} \quad=$ second degree zonal harmonic coefficient of the potential of gravitation

$\mathrm{km}=$ kilometers

$\lambda \quad=$ longitude

$\mathrm{m} \quad=$ meters

$\mathrm{M} \quad=$ mass of the world

\footnotetext{
* Aerospace Engineer, Flight Simulation and Software Branch, Mail Stop 125B, Senior Member AIAA.
} 


$\begin{array}{ll}\mathrm{P}_{\mathrm{n}, \mathrm{m}} & =\text { associated Legendre polynomial of degree } \mathrm{n} \text { and order } \mathrm{m} \\ \mathrm{P}_{\mathrm{n}} & =\text { Legendre polynomial of degree } \mathrm{n} \\ \mu & =\text { gravitational constant of the world } \\ |\mathbf{r}|, \mathrm{r} & =\text { the magnitude of the radius vector from the object to the center of the world } \\ \hat{\mathbf{r}} & =\text { the unit vector of an object's position relative to the world's center } \\ \mathrm{S} & =\text { seconds } \\ \mathrm{S}_{\mathrm{n}, \mathrm{m}} & =\text { spherical harmonic coefficient (degree } \mathrm{n} \text {, order } \mathrm{m} \text { ) of the gravitational potential } \\ \mathrm{S} / \mathrm{C} & =\text { Rotating sphere Earth model with constant gravitation } \\ \mathrm{S} / \mathrm{PM} & =\text { Rotating sphere Earth model with point-mass gravitation } \\ \mathrm{S} / \mathrm{SE} & =\text { Rotating sphere Earth model with symmetric ellipsoid gravitation } \\ \mathrm{SNR} / \mathrm{C} & =\text { Non-rotating sphere Earth model with constant gravity } \\ \mathrm{SNR} / \mathrm{FA} & =\text { Non-rotating sphere Earth model with free-air reduction gravity } \\ \mathrm{V} & =\text { potential of gravitation } \\ \mathrm{WGS} 84 & =\text { World Geodetic System } 1984 \\ \mathrm{x}, \mathrm{y}, \mathrm{z} & =\text { Earth-fixed Cartesian coordinates }\end{array}$

\section{Introduction}

A erospace simulations can model worlds, such as the Earth, with differing levels of fidelity. The simulation may represent the world as a plane, a sphere, an ellipsoid, or a high-order closed surface. The world model may or may not include the rotation of the world. Likewise, the simulation can model gravity or the gravitation component, as appropriate, using models of differing fidelities; examples include constant gravity, constant gravitation, free-air reduction, point-mass gravitation, and a spherical-harmonic expansion of the geopotential. The user may select lower fidelity models based on computational limits, a need for simplified analysis, or comparison to other data. In many simulation products, the world and gravity/gravitation are modeled independently, allowing users to select any combination of world and gravity/gravitation model. Independently selecting the gravity/gravitation and world model does not necessarily result in a combined fidelity equivalent to the fidelity of the individual models. Some pairs produce degraded fidelity. ${ }^{1}$

This paper examines the actual performance of world and gravity/gravitation pairs in a simulation using the Earth. Performance is compared against a rotating WGS-84 ellipsoid of the Earth whose density is symmetric about the polar axis. The paper applies the selected pairs to both a point-mass without drag and a large civilian aircraft. The point-mass without drag isolates the effect of various world and gravity/gravitation pairs on the surface relative state of the object. The large civilian aircraft demonstrates how that influence changes when other forces act on the object. The investigation included a limited number of pairs based on prior analytical work Reference 1; the pairs are presented in section II.C. The next section provides a brief overview of the world and gravity/gravitation models. More detailed treatment of these models is found in Reference 1.

\section{The Models}

\section{A. World Models}

World models simulate the motion of the world and provide an idealized approximation of its shape. A world is any body whose shape is roughly spherical under the influence of its self-gravity. Since this paper investigates choices for combining the world model with a gravity or gravitation model, only the world's motion relative to its center of mass, i.e. its rotation, is relevant. The Earth has a dominant rotation about one axis; the rotation axis will be the z-axis in an Earth-fixed, Cartesian coordinate system. This paper focuses on the sphere and ellipsoid shape models that are commonly used in modeling the Earth. Higher order shapes can provide improved modeling for the Earth but are not in common use among vehicle simulation products. This paper does not examine modeling the Earth as a plane, i.e. the "flat Earth" model. The "flat Earth" model maintains surface fidelity over small distances. Earth's gravity doesn't change significantly over such distances; thus, a constant gravity model or the free air reduction model (see section II.B) can provide accurate gravity values by using the local surface gravity as a reference. ${ }^{1}$ The modeling of gravity or gravitation becomes important only as one travels large distances over the Earth.

The sphere model is a good approximation for bodies whose shape is determined by its self-gravity. It represents the Earth as a sphere of constant radius. Possible choices for the radius include: the equatorial radius, the average of the equatorial and polar radii, the mean radius, the radius of a sphere of equal surface area to the reference ellipsoid, and the radius of a sphere of equal volume to the reference ellipsoid. For this investigation, the radius of equal sur- 
face area to the ellipsoid was used; Reference 1 demonstrated that this radius minimizes the error in the mean surface gravity.

The ellipsoid model represents the Earth as an ellipse of revolution about its semi-minor axis (i.e. an oblate spheroid). The ellipsoid is defined as an equipotential surface of the potential of gravity. ${ }^{2}$ Four parameters define its geometry. This investigation uses the four parameters of the World Geodetic System 1984 (WGS84). ${ }^{2}$ WGS84's parameters are the semi-major axis (a), the Earth's gravitational constant (GM), the flattening (f), and the angular velocity $(\omega)$ of the Earth.

Both the sphere and ellipsoid can model the rotation of the world, and modeling rotation will increase the overall fidelity of those models. But, one can also set the rotation to zero for simplicity. Disabling rotation removes the centrifugal acceleration and Coriolis acceleration terms. The centrifugal acceleration is a function of position. Its value near the surface is approximately fixed for a given latitude. That value is small on the Earth; the mean is $0.23 \%$ of gravity. But, the Coriolis acceleration is a function of the object's velocity and remains minute only for low speed vehicles (e.g. subsonic). Thus, non-rotating worlds are viewed as appropriate for simulations of lowspeed vehicles whose travel typically is limited to the world's surface. Rotation also directly impacts how gravity is modeled. Gravity is the combination of gravitation and centrifugal acceleration. ${ }^{3}$ If rotation is turned off, a surface interacting simulation should model gravity in order to reproduce the free fall of objects as observed from the Earth surface. Modeling gravitation without modeling rotation will produce slightly larger free fall at locations away from the poles (where the centrifugal acceleration is zero). If rotation is modeled, then the simulation should not use a gravity model; doing so will exaggerate the affect of the centrifugal acceleration. Gravitation models should be used when rotation is modeled.

\section{B. Gravity and Gravitation Models}

This paper will examine two gravity models and three gravitation models. The need for both gravity and gravitation models is explained in the previous section. The gravity models are constant gravity and the free air reduction. The gravitation models are constant gravitation, point-mass gravitation, gravitation of a symmetric ellipsoid. The gravitation of the symmetric ellipsoid is approximated using a spherical harmonic expansion of the gravitational potential.

\section{Constant Gravity and Gravitation}

The constant gravity and gravitation models use a single value for gravity or gravitation regardless of the vehicle's location. Gravity acts along the surface normal and gravitation acts along the radius between the vehicle and the center of the Earth. The gravity constant selected for this model is standard gravity, $9.80665 \mathrm{~m} / \mathrm{s}^{2}\left(32.174 \mathrm{ft} / \mathrm{s}^{2}\right)$. Standard gravity is a standard unit of acceleration ("g") and not a physical property of the Earth. However, its error profile against the theoretical surface gravity shows improved fidelity over the mean gravity (which is a physical property). ${ }^{1}$ The gravitation constant selected for this model is the mean gravitation, $9.82023 \mathrm{~m} / \mathrm{s}^{2}\left(32.219 \mathrm{ft} / \mathrm{s}^{2}\right)$.

\section{Free Air Reduction}

The free air reduction is a gravity model that accounts for the decrease in gravity with height. The free air reduction is a linear approximation for the vertical gradient of gravity in the neighborhood of the reference geoid; the linear change in gravity with height is approximately $3.086 \times 10^{-6} \mathrm{~s}^{-2} .4$ The resulting gravity formula is:

$$
g=g^{s}+3.086 \times 10^{-6} h
$$

where $\mathrm{g}^{\mathrm{s}}$ is the reference gravity on the surface and $\mathrm{h}$ is the altitude. The free air reduction is valid within the vicinity of the surface. This model has degraded fidelity above $15.9 \mathrm{~km}(\sim 52,000 \mathrm{ft}){ }^{1}$

\section{Point Mass Gravitation}

According to Newton's universal law of gravitation, the attracting force between two masses is inversely proportional to the square of the distance between them and directly proportional to the product of their masses. The gravitation exerted by a world on an object depends on the distribution of its mass. A spherically symmetric mass is a good first approximation. A spherically symmetric mass can be treated as if all of its mass where located at its center, i.e. a point mass. One can compute the gravitational acceleration imparted by a point-mass using Equation 2:

\section{Symmetric Ellipsoid Gravitation}

$$
\vec{\gamma}=-\frac{G M}{|\mathbf{r}|^{2}} \hat{\mathbf{r}}
$$

The Earth is not a spherically symmetric mass. The Earth bulges at the equator as a consequence of its rotation. The density of the Earth also varies from location to location. The landmass topography and the oceans are visible reminders that the Earth is not a symmetric mass. The variability of the Earth's mass is modeled by expressing the 
world's gravitational potential as a spherical harmonic expansion. The spherical harmonic expansion of gravitational potential has the form: ${ }^{2,4}$

$$
V\left(r, \phi_{g}, \lambda\right)=\frac{G M}{r}\left(1+\sum_{n=2}^{\infty} \sum_{m=0}^{n}\left(\frac{a}{r}\right)^{n} P_{n, m}\left(\sin \phi_{g}\right)\left[C_{n, m} \cos (m \lambda)+S_{n, m} \sin (m \lambda)\right]\right)
$$

where

$\mathrm{V} \quad$ is the gravitational potential at geocentric spherical coordinates: radius (r), latitude $\left(\phi_{\mathrm{g}}\right)$, and longitude $(\lambda)$

$\phi_{\mathrm{g}} \quad$ is the geocentric latitude ${ }^{\Uparrow}$

$\mathrm{r} \quad$ is the geocentric radius

$\lambda \quad$ is the geocentric longitude

$\mathrm{G} \quad$ is the universal gravitational constant

$\mathrm{M} \quad$ is the mass of the world

a is the semi-major axis of the reference ellipsoid

$\mathrm{n} \quad$ is the degree of the harmonic term

$\mathrm{m} \quad$ is the order of the harmonic term

$\mathrm{C}_{\mathrm{n}, \mathrm{m}}, \mathrm{S}_{\mathrm{n}, \mathrm{m}}$ are the harmonic coefficients

$\mathrm{P}_{\mathrm{n}, \mathrm{m}}\left(\sin \phi_{\mathrm{g}}\right)$ is the associated Legendre polynomial of order $\mathrm{n}$ and degree $\mathrm{m}$ with an argument of $\sin \left(\phi_{\mathrm{g}}\right)$. See Ref. 4 for a definition of the Legendre polynomials.

Equation 3 can be simplified significantly if one assumes that the Earth's mass is symmetric about its polar axis (i.e. the minor axis) and its equator. Symmetry about the polar axis is accomplished by setting the order (m) to zero. The harmonics with order $\mathrm{m}=0$ are called zonal harmonics and capture mass distribution effects which do not depend on longitude, i.e. the effect has rotational symmetric about the polar axis. The remaining harmonics $(\mathrm{m} \neq 0)$ capture mass distribution effects that depend on longitude (solely or in combination with latitude). Setting $\mathrm{m}=0$ reduces Equation 3 to:

$$
V\left(r, \phi_{g}\right)=\frac{G M}{r}\left(1+\sum_{n=2}^{\infty}\left(\frac{a}{r}\right)^{n} C_{n} P_{n}\left(\sin \phi_{g}\right)\right)
$$

where $C_{n}=C_{n, 0}$ and $P_{n}\left(\sin \phi_{g}\right)=P_{n, 0}\left(\sin \phi_{g}\right)$. This formulation still allows mass distribution to differ between the southern and northern hemispheres. To impose symmetry about the equator, the odd zonal harmonics $\left(\mathrm{C}_{\mathrm{n}}\right.$ where $\mathrm{n}$ is odd) are set to zero.

To obtain the gravitation at a given location, one takes the gradient of the gravitational potential (Equation 4). Equations 5 and 6 show the resulting gravitation equations when expanded to degree $(n)=$ four:

$$
\begin{aligned}
& \gamma_{r}=\frac{\partial V}{\partial r}=-\frac{\mu}{r^{2}}\left[1+\frac{3}{2}\left(\frac{a}{r}\right)^{2} C_{2}\left(3 \sin ^{2} \phi_{g}-1\right)+\frac{5}{8}\left(\frac{a}{r}\right)^{4} C_{4}\left(35 \sin ^{4} \phi_{g}-30 \sin ^{2} \phi_{g}+3\right)\right] \\
& \gamma_{\phi_{g}}=\frac{1}{r} \frac{\partial V}{\partial \phi_{g}}=\cos \phi_{g} \sin \phi_{g} \frac{\mu}{r^{2}}\left(\frac{a}{r}\right)^{2}\left[3 C_{2}+\frac{1}{2}\left(\frac{a}{r}\right)^{2} C_{4}\left(35 \sin ^{2} \phi_{g}-15\right)\right]
\end{aligned}
$$

The results in this study use equations expanded to degree $(n)=8$ because the resulting equations match WGS 84 published gravitation values to ten significant digits. ${ }^{1}$

The values of the even harmonic coefficients $\left(C_{n}\right.$ where $n$ is even) remain to be defined. Moritz shows that the second degree zonal harmonic $\left(\mathrm{C}_{2}\right)$ is a function of the world's mass, equatorial radius, and moments of inertia (Equation 17). ${ }^{4,5}$ All subsequent zonal harmonics are functions of $\mathrm{C}_{2}$ (Equation 7).

$$
C_{2}=\frac{I_{z Z}-I_{x X}}{M a^{2}} \quad, \quad C_{2 n}=(-1)^{n} \frac{3 e^{2 n}}{(2 n+1)(2 n+3)}\left(1-n-5 n \frac{C_{2}}{e^{2}}\right)
$$

\footnotetext{
"This paper defines geocentric latitude as it is used in the WGS84 model. Geocentric latitude is the angle between the equatorial plane and the radius from the center of the world to a point. The point may be on or above the surface of the reference ellipsoid.
} 
where e is the eccentricity of the Earth. Since the world's mass properties cannot be measured directly, $\mathrm{C}_{2}$ is empirically derived. For the Earth, $\mathrm{C}_{2}=-0.00108262982131$ and is computed by multiplying the WGS84 normalized coefficient $\left(\mathrm{C}_{2,0}\right)$ with the normalization function $\sqrt{2 n+1}$. $^{2}$

\section{Paring World and Gravity/Gravitation Models}

The world and gravity/gravitation models can be combined to produce ten pairs. This is less than the number of possible permutations because some pairs are eliminated due to incompatible physics. As explained in section II.A, gravity models should only be paired with non-rotating world models because gravity models include a centrifugal acceleration value that is already included in the equations of motion for rotating world models. Likewise, gravitation models should only be used with rotating world models; they will produce higher than observed free-fall accelerations in non-rotating world models because non-rotating world models lack centrifugal acceleration. The remaining ten pairs are:

- Rotating sphere Earth with symmetric ellipsoid gravitation (S/SE)

- Rotating ellipsoid with point-mass gravity (E/PM)

- Non-rotating sphere Earth with global gravity constant (SNR/C) - limited to $1.9 \mathrm{~km}$

- Non-rotating ellipsoid Earth with global gravity constant (ENR/C) - limited to $1.9 \mathrm{~km}$

- Non-rotating sphere Earth with free air reduction (SNR/FA) - limited to $15.9 \mathrm{~km}$

- Non-rotating ellipsoid Earth with free air reduction (ENR/FA) - limited to $15.9 \mathrm{~km}$

- Rotating sphere Earth with global gravitation constant (S/C) - limited to $7.4 \mathrm{~km}$

- Rotating ellipsoid Earth with global gravitation constant (E/C) - limited to $7.4 \mathrm{~km}$

- Rotating sphere Earth with point mass gravitation (S/PM)

- Rotating ellipsoid Earth with geopotential model for a uniform ellipsoid (E/SE)

Because all pairs will produce gravity to a surface observer, the pairs will be called world-gravity pairs. Each world-gravity pair is listed with a short-hand acronym that is used in later text and charts. The pairs are listed in approximate order of their gravity fidelity near the surface. ${ }^{1}$ Analytical comparison of surface gravity performance demonstrates that the first two pairs produce lower fidelity results than the individual models would suggest. However, they have been included to evaluate whether poor surface gravity modeling translates into equally poor dynamics performance. Note that the symmetric ellipsoid gravitation can only be paired with the rotating sphere Earth if the gravitation model uses an independent set of physical properties (i.e. moments of inertia, eccentricity, and equatorial radius) that derive from the ellipsoid model of the Earth. If the gravitation model shared the same physical properties with sphere model (e.g. eccentricity equals zero), the model would reduce to the point-mass representation. The last pair (E/SE) matches the theoretical surface gravity of a rotating Earth in hydrostatic equilibrium and is used as the truth state against which the dynamics performance of the other pairs is compared. Some world-gravity pairs are listed with an upper altitude limit. Beyond this limit, gravity fidelity of this world-gravity pair degrades beyond the elimination of higher order effects. ' (Higher order effects are the centrifugal acceleration and the nonspherical distribution of the Earth's mass).

\section{Method}

This paper uses two scenarios to evaluate the surface-relative dynamics performance of vehicle models under each pair of world and gravity/gravitation model. The first scenario is a mass without drag in a low-altitude circular orbit. The second scenario is a large subsonic commercial transport aircraft starting at the same altitude as the first scenario. The aircraft runs without an active control system ("open-loop"). The first scenario is designed to isolate the impact of the world-gravity pairs on the dynamics of the vehicle relative to the surface. However, to remain aloft long enough to traverse a hemisphere for at least half the world-gravity pairs, the vehicle must travel at orbital speeds that approach Mach 25. This scenario is contrived since no man-made vehicle can make this flight. The second scenario represents a more realistic near-surface scenario. In this scenario, however, gravitation is only one of the forces that act on the vehicle, and it is largely counteracted by the aerodynamic lift on the vehicle. So the impact of gravity values on vehicle dynamics may be less significant. 
Table 1 Initial Conditions for Simulation Scenarios

\begin{tabular}{|c|c|c|c|}
\hline & & Scenario 1: Low-altitude Orbit & Scenario 2: Subsonic Transport \\
\hline \multicolumn{2}{|l|}{ geodetic latitude } & \multicolumn{2}{|c|}{0 degrees } \\
\hline \multicolumn{2}{|l|}{ longitude } & \multicolumn{2}{|c|}{32.45 degrees } \\
\hline \multicolumn{2}{|l|}{ altitude } & \multicolumn{2}{|c|}{$24278.2152231 \mathrm{ft}(7.4 \mathrm{~km})$} \\
\hline \multicolumn{2}{|l|}{ world-relative velocity } & {$[25890.75846025,0.0,0.0] \mathrm{ft} / \mathrm{s}$} & {$[674.8311157,0.0,0.0] \mathrm{ft} / \mathrm{s}$} \\
\hline \multirow{3}{*}{ world-relative orientation } & $\phi$ & 0 degrees & $\sim 0$ degrees $^{\mathrm{a}}$ \\
\hline & $\theta$ & 0 degrees & 2.3586 to 2.3850 degrees $^{b}$ \\
\hline & $\psi$ & 0 degrees & $\sim 0$ degrees $^{\mathrm{a}}$ \\
\hline \multirow{3}{*}{$\begin{array}{l}\text { world-relative angular velocity } \\
\text { (in body coordinates) }\end{array}$} & $\mathrm{P}$ & $0 \mathrm{deg} / \mathrm{sec}$ & $\sim 0 \mathrm{deg} / \mathrm{sec}^{\mathrm{a}}$ \\
\hline & Q & $0 \mathrm{deg} / \mathrm{sec}$ & -0.0018477 to $-0.0018580 \mathrm{deg} / \mathrm{sec}$ \\
\hline & $\mathrm{R}$ & $0 \mathrm{deg} / \mathrm{sec}$ & $\sim 0 \mathrm{deg} / \mathrm{sec}^{\mathrm{a}}$ \\
\hline \multicolumn{2}{|l|}{ elapsed time } & 90 minutes & 12 hours \\
\hline
\end{tabular}

Table 1 presents the initial conditions for each scenario. In both cases, the vehicles start on the equator at coordinates $0^{\circ}$ geodetic latitude and $32.45^{\circ}$ longitude. (This puts the vehicle near the Ugandan city of Entebbe.) Both vehicles face and travel north. The predominant variation in Earth's gravity occurs as one travels from the equator to either pole. The scenarios aim to travel along this path, providing the worst case difference among the worldgravity pairs. Both scenarios begin at an altitude of $7.4 \mathrm{~km}(24,278 \mathrm{ft})$. This is at the upper limit of "acceptable" gravity performance for the constant gravitation model (either $\mathrm{E} / \mathrm{C}$ or $\mathrm{S} / \mathrm{C}$ ) and is above the recommended altitude for the constant gravity model (either ENR/C and SNR/C).

In both scenarios, the initial velocity is defined as a world-relative value. Thus, an observer on the surface of the world model will measure the same initial velocity for the vehicle, whether the world rotates or not. However, the inertial velocity of the vehicle differs between simulations using a rotating world model and simulations using a non-rotating world model. The simulations using a rotating world model will impart an additional inertial velocity in the easterly direction so that the vehicle has no net East-West velocity relative to the surface. The purpose of the investigation is to compare the surface-relative dynamics of vehicles under different world-gravity pairs of world and gravity/gravitation models. Thus, the surface-relative state of the vehicle is the same for all runs; the inertial state may differ. This is especially important for the aircraft scenario in which the vehicle experiences aerodynamic forces. The atmosphere is assumed to rotate with the world. Thus, the vehicle must also rotate with the world to prevent large sideslip. In the orbital scenario, the speed of the vehicle is set to create a circular orbit under the rotating sphere Earth model. A circular orbit should provide broad latitude coverage without the vehicle falling below the Earth's surface. The orbit speed was based on the rotating sphere model for two reasons. First, the starting position is a shorter distance from the Earth center in the sphere model than the ellipsoid model. Thus, the sphere model requires a higher speed for a circular orbit than the ellipsoid model. As a result, the orbit under the ellipsoid Earth model will be slightly elliptical, but should still remain in the atmosphere. Second, only the rotating models are paired with the point-mass gravitation model that can produce a circular orbit using the Earth's published gravitational constant. Since the vehicle on the rotating model will have an inertial velocity that has an easterly component upon initialization, the initial north velocity component was computed by subtracting the easterly component from the required speed for a circular orbit, $\sim 25,921 \mathrm{ft} / \mathrm{s}$. The resulting north velocity is $\sim 25,891 \mathrm{ft} / \mathrm{s}$. For the aircraft scenario, the speed of the vehicle is set to approximately $674 \mathrm{ft} / \mathrm{s}$ ( 280 knots indicated air speed $)$. At this speed, the aircraft travels from the equator to pole in about 13.5 hours.

Since gravity is the only acting force in the orbital scenario and it is not a function of vehicle orientation, the initial vehicle orientation and angular velocity can be arbitrarily set. The scenario can start without running a solver to balance body forces and moments. In the aircraft scenario, the vehicle generates engine thrust and aerodynamics forces. The scenario is designed to start the vehicle in straight and level flight. A solver is run to determine the vehicle's world-relative orientation and angular velocity that produces straight and level flight, i.e. a net world-relative acceleration of zero and maintaining the vehicle's world-relative orientation as it traverses the curved surface of the Earth. Since the aircraft is initialized to face and travel due north, the Euler angles $\phi$ and $\psi$ and the angular velocities $\mathrm{P}$ and $\mathrm{R}$ are zero. Only the Euler angle $\theta$ and the angular velocity $\mathrm{Q}$ require non-zero values to establish straight and level flight. 
The elapsed time for the orbital scenario was 90 minutes. This time allows for a complete orbit of the vehicle; the orbital period is $\sim 85$ minutes. The elapsed time for the aircraft scenario was 12 hours. During this time the vehicle travels from the equator to $\sim 80^{\circ}$ geodetic latitude. The simulations were constructed and operated using the Langley Standard Real-Time Simulation in C++ (LaSRS++). ${ }^{6}$ The simulations were run using the default secondorder integrators in LaSRS++. The orbital scenario was run at $500 \mathrm{~Hz}$ to reduce the influence of integration error. Recent internal tests have verified that this configuration has equivalent integration error to Johnson Space Center's Trick simulation using $4^{\text {th }}$ order Runge-Kutta integrators at $30 \mathrm{~Hz}$. The aircraft scenario was run at $50 \mathrm{~Hz}$, which is the verified and validated iteration rate for the aircraft model that was used.

\section{Results}

Figure 1 through Figure 9 present the results of the simulations. Unfortunately, the results of some world-gravity pairs are nearly identical and the data lines for one or more world-gravity pairs are hidden behind others. Notes have been added to the charts to highlight these overlaps. The figures provide the time evolution of the surface relative position of the vehicle and the magnitude of the gravity/gravitation model output. The surface relative position is provided as geodetic latitude, geodetic longitude, and geodetic altitude (i.e. height above the surface as measured along the surface normal). The magnitude of the gravitational acceleration is the magnitude of the acceleration vector that the gravity or gravitational model computes. This vector is the acceleration that the world-gravity pair exerts on the vehicle as observed in an inertial reference frame; this is how the model output is treated in the LaSRS++ simulation. For the non-rotating world-gravity pairs, this vector is gravity (i.e. includes a centrifugal acceleration factor). For the rotating world-gravity pairs, the vector is purely gravitation. The simulation results were further reduced to a series of position error vectors. To compute the position error, the surface relative position of each run was transformed into an Earth centered vector using equations 4-14 and 4-15 in Ref. 2. These equations relocate the surface relative positions onto the ellipsoid Earth so that all surface relative positions are referenced to the same shape. The position vector for a world-gravity pair is subtracted by the position vector of the reference worldgravity pair, E/SE. The result is the position error relative to the reference pair (E/SE). The maximum magnitude of the position error for each world-gravity pair is presented in Table 2 and Table 3 . The tables are ordered by increasing error so the ordering of the world-gravity pairs differs between the two tables.

Table 2 Orbital Scenario - Maximum Distance Error

\begin{tabular}{|l|r|}
\hline $\begin{array}{l}\text { World- } \\
\text { gravity } \\
\text { pair }\end{array}$ & $\begin{array}{c}\text { Max distance error } \\
\text { (nautical miles) }\end{array}$ \\
\hline E/PM & 17.831551 \\
\hline S/PM & 63.579954 \\
\hline S/SE & 81.329253 \\
\hline E/C & 87.711123 \\
\hline S/C & 95.542104 \\
\hline ENR/C & 1272.513727 \\
\hline ENR/FA & 1275.171682 \\
\hline SNR/FA & 1276.120095 \\
\hline SNR/C & 1276.120725 \\
\hline
\end{tabular}

Table 3 Aircraft Scenario - Maximum Distance Error

\begin{tabular}{|l|r|}
\hline $\begin{array}{l}\text { World- } \\
\text { gravity } \\
\text { pair }\end{array}$ & $\begin{array}{c}\text { Max distance error } \\
\text { (nautical miles) }\end{array}$ \\
\hline ENR/FA & 23.314419 \\
\hline ENR/C & 23.314427 \\
\hline SNR/FA & 23.950602 \\
\hline SNR/C & 23.950602 \\
\hline E/C & 50.616232 \\
\hline E/PM & 50.769952 \\
\hline S/C & 57.447983 \\
\hline S/PM & 57.615892 \\
\hline S/SE & 97.953584 \\
\hline
\end{tabular}

\section{A. The Orbital Scenario}

Figure 3 shows that all vehicle trajectories remain in the atmosphere. The orbital scenario succeeds in producing near-surface trajectories. However, seven of the ten world-gravity pairs crash into the surface. Thus, caution is warranted when examining the maximum distance errors in Table 2. The table contains the maximum errors across the full run of the simulation. Results for world-gravity pairs are not physically valid after they impact the surface. Figure 3 and Figure 4 discard the invalid data to improve readability of the chart. Complete runs appear in Figure 1 and Figure 2 because the data remains tightly clustered, and the invalid data provides insight into the results in Table 2. Table 2 uses the complete run for all world-gravity pairs in order to provide a consistent and meaningful comparison. Ending the position error computation for a world-gravity pair upon surface impact would have stopped the error computation early in the simulation before large position errors appear in the world-gravity pairs that complete 
the run. The resulting maximum errors would be artificially skewed in favor of the crashed scenarios. If one were to judge the world-gravity pairs based first on time before crashing and then on error position, the order becomes:

1) $\mathrm{E} / \mathrm{PM}$

2) $\mathrm{S} / \mathrm{PM}$

3) $\mathrm{E} / \mathrm{C}$

4) $\mathrm{ENR} / \mathrm{FA}$

5) $\mathrm{S} / \mathrm{SE}$

6) $\mathrm{ENR} / \mathrm{C}$

7) $\mathrm{S} / \mathrm{C}$

8) $\mathrm{SNR} / \mathrm{FA}$

9) $\mathrm{SNR} / \mathrm{C}$

All of the world-gravity pairs with a non-rotating Earth model crash. This is expected since the North velocity was chosen to produce an orbital velocity with the rotating sphere. Vehicles in the non-rotating Earth are not given an inertial Easterly velocity to maintain longitude and therefore have a lower inertial velocity than required to maintain orbit. Three of the rotating cases also crash: E/C, S/SE, and S/C. The two sphere cases can be explained by comparing them against the S/PM world-gravity pair. The velocity was initialized to produce a circular orbit for $\mathrm{S} / \mathrm{PM}$, and this is evidenced in Figure 3 where the S/PM simulation maintains a constant altitude. The S/SE and S/C world-gravity pairs crash because they produce gravitational accelerations greater than S/PM (see Figure 4) and cause the vehicle to immediately de-orbit. The $\mathrm{E} / \mathrm{C}$ world-gravity pair manages to remain aloft for a considerable time because it is helped by two factors. The first is that the vehicle inertial position in the ellipsoid Earth begins about $14.5 \mathrm{~km}$ further from the Earth's center than its inertial position under the sphere model. Thus, the initial velocity is greater than necessary for a circular orbit in the ellipsoid Earth. The constant gravitation acceleration will thus take longer to drag the vehicle to the surface. The second factor is that the surface initially slopes away from the vehicle at the same time; without the elliptical shape of the surface, the vehicle would crash before it approached the Northern artic. The constant gravitation model, however, eventually brings the vehicle to the surface short of performing a full orbit.

The vehicle's trajectory varies widely depending on the world-gravity pair. In this scenario, the world model is equally important to the gravity/gravitation model. The initial conditions are defined relative to the world and differences in world model produce orbits with different characteristics. Vehicles in the rotating world model gain an easterly velocity to maintain longitude; the easterly velocity decreases the inclination of the orbit. The non-rotating pairs start with an orbit inclination of $90^{\circ}$ (polar). The rotating pairs start with an orbit inclination of $86.627^{\circ}$. The difference in inclination is $3.373^{\circ}$. The difference in inclination is a major cause of latitude error in the non-rotating worlds. Indeed, the maximum latitude error for the non-rotating pairs ranges from $2.86^{\circ}$ to $3.39^{\circ}$ and occurs near the South Pole. Data for the orbit scenario was collected at an interval of one minute. The sampling rate causes the reported error to be less than the difference in inclination. Orbit shape differs between the ellipsoid and sphere models. Vehicles in the ellipsoid world start $14.5 \mathrm{~km}$ further from the Earth's center than vehicles in the sphere world. The initial velocity, which produces a circular orbit in the sphere world, will produce an elliptical orbit in the ellipsoid world. Since the reference pair uses the ellipsoid Earth, the difference between the apoapsis and periapsis of its orbit represents the expected error that the sphere pairs will produce as they cross the equator on the opposite side of the Earth; the difference is $\sim 24,700 \mathrm{ft}(7.5 \mathrm{~km})$ in this scenario. Sphere models will incur additional errors at the poles because the orbit shape is independent of surface shape. The pole in the ellipsoid Earth is $\sim 47,000 \mathrm{ft}(14.3$ $\mathrm{km})$ closer to the center than the pole of the sphere Earth. The maximum error for S/PM is $71,500 \mathrm{ft}(21.8 \mathrm{~km})$ and occurs near the North Pole. Not only is the shape of the orbit different between the sphere and ellipsoid Earth models, but its period is also different. The circular orbit has a faster period and the vehicles in the sphere model will lead those in the ellipsoid model. Between E/PM and S/PM, the period differs by 17 seconds. E/SE produces a slightly faster period than E/PM, so the period difference with S/PM decreases to around 13 seconds. In that time the vehicle travels $\sim 55.5$ nautical miles. The four second period difference between E/PM and E/SE represents a distance error of $\sim 17$ nautical miles. These distance error are approximately equal to the maximum distance errors in Table 2; thus, differences in orbital period are the primary contributor of distance error among the rotating models. Because the orbit is nearly polar, the period difference is also the main cause of errors in latitude.

Once propagating through time, only the gravity/gravitation model influences the path of the vehicle in inertial space. The orbits are fixed in inertial space. But, the world model determines the translation of the inertial positions into surface relative coordinates. Thus, the rotation of the Earth will continually change the longitude underneath the orbit. Non-rotating world models immediately incur a longitude error equal to the product of the Earth rotation and the elapsed time. The vehicle's longitude versus time (Figure 2) shows this division between the rotating and non-rotating worlds. Under a non-rotating world, the vehicle stays at $32.45^{\circ}$ longitude or its compliment $-147.55^{\circ}$. 
The rotating worlds turn $21.22^{\circ}$ during the orbital period of $\sim 85$ minutes. The vehicles operating in a rotating world, therefore, find themselves at $\sim 11^{\circ}$ latitude when they complete an orbit. The $21.22^{\circ}$ difference in longitude represents a distance of $\sim 1278$ nautical miles near the equator (at altitude); this approximately matches the maximum distance error for the non-rotating worlds in Table 2. Thus, the longitude error is the major contributor to position error for the non-rotating worlds.

In the orbital case, inertial observation reveals the surface-relative differences among the world-gravity pairs. Gravitation (gravity) is a significant determinant of location error; but the fidelity of gravity/gravitation model in inertial space and not at heights above the Earth is the important factor. Though the scenario produce vehicles trajectories that remain near the surface, a drag-less vehicle traveling at such high speeds is no longer bound to the Earth. The scenario falls outside the notion of a surface-interacting simulation. This attempt to craft a surfaceinteracting scenario under the influence of gravity alone did not succeed. The scenario will not provide much illumination on the affect that the gravity fidelity of the world-gravity pairs have on surface-relative dynamics. The scenario reinforces current practice for orbital simulations: do not disable rotation of the Earth and do not use constant gravitation models. What this scenario does corroborate is the inappropriateness of using a geopotential model like the symmetric ellipsoid gravitation model with the rotating sphere. ${ }^{1}$ The S/SE pair crashed into the Earth and generally performed worse than the lower fidelity point-mass gravitation model in the S/PM pair. Though analysis tags the E/PM pair with poor gravity fidelity, the pair emerges as the best reasonable lower-fidelity alternative to the reference pair E/SE. The E/PM pair benefits from the fact that this scenario isn't influenced by gravity at height above surface but by gravitation in inertial space. In a sense, this scenario does lend itself to independent selection of gravitation and world model.

\section{B. Aircraft Scenario}

This scenario differs substantially from the orbital scenario. The vehicle is traveling at almost $1 / 40^{\text {th }}$ the speed. The vehicle generates forces that counteract the gravitational acceleration, and those forces are functions of Earthrelative quantities (e.g. altitude, wind-relative velocity). The scenario is also run for twelve hours. Table 3 shows that this scenario produces nearly opposite results from the orbital case. The best performing world-gravity pairs use non-rotating models of the Earth! The culprit is centrifugal acceleration and it works against the rotating worldgravity pairs in this scenario. The centrifugal acceleration is perpendicular to the axis of rotation and, except, at the equator is not normal to the surface; thus it produces a tangential acceleration. The ellipsoid Earth is defined as an equipotential surface of the gravity potential. In other words, the Earth is shaped such that the gravitational acceleration has a tangential component that counterbalances the tangential component of centrifugal acceleration so that the resulting gravity vector always remains normal to the surface. This balance is correctly modeled in the reference world-gravity pair, the ellipsoid Earth paired with the symmetric ellipsoid gravitation model. However, this balance is disrupted in all other world-gravity pairs with a rotating world. For the rotating sphere, the constant and pointmass gravitation models produce gravitation that is always normal to the surface. There is no tangential component of gravitation to counteract the tangential component of centrifugal acceleration. The residual tangential component of gravity is entirely due to centrifugal acceleration; its mean magnitude over the surface of the Earth is 0.01129 $\mathrm{m} / \mathrm{s}^{2}\left(0.037 \mathrm{ft} / \mathrm{s}^{2}\right)$, directed toward the equator. ${ }^{1}$ The symmetric ellipsoid gravitation model does not produce gravitation that is normal to the surface of the sphere at most locations. It contributes to the residual tangential component of gravity and nearly doubles the mean value on the surface to $0.02194 \mathrm{~m} / \mathrm{s}^{2}\left(0.0712 \mathrm{ft} / \mathrm{s}^{2}\right)$ toward the equator. On the ellipsoid Earth, the constant and point-mass gravitation models usually not normal to the surface, except at the equator and pole. The models leave a tangential component of gravitation that overcompensates for the tangential component of centrifugal acceleration. The result is residual tangential component of gravity whose surface mean is $0.01073 \mathrm{~m} / \mathrm{s}^{2}\left(0.0352 \mathrm{ft} / \mathrm{s}^{2}\right)$ and directed toward the pole. The non-rotating world models do not exhibit a centrifugal acceleration. The gravity models that they are paired with do contain a centrifugal acceleration factor to compensate but the entire value is applied to the surface normal and has no tangential component. Thus, the nonrotating worlds produce no residual tangential gravity and, therefore, more closely match the gravity behavior of the E/SE world-gravity pair. Figure 6 corroborates the analysis. Because the residual tangential gravity tugs them toward the equator, the S/PM and S/C world-gravity pairs are $0.95^{\circ}$ lower in latitude at the end of the run and the S/SE world-gravity pair is $1.62^{\circ}$ lower. The $\mathrm{E} / \mathrm{C}$ and $\mathrm{E} / \mathrm{PM}$ world-gravity pairs are $0.84^{\circ}$ higher, being tugged north by the residual tangential gravity. Among these pairs, the magnitudes of the latitude errors are similar in proportion to the magnitude of the residual tangential gravity; e.g., the latitude error for E/PM is about the same magnitude and opposite in direction to the S/PM latitude error. The residual tangential acceleration of gravity also appears to cause the altitude variations in Figure 8. The rotating sphere pairs drop in altitude and the E/C and E/PM world-gravity pairs climb in altitude. The altitude changes peak in the mid upper latitudes where the maximum tangential gravity occurs. At the upper latitude, the tangential gravity diminishes and the vehicles begin their return to their starting 
altitude. The S/C and S/PM world-gravity pairs hit a maximum difference of $\sim 860$ feet lower. The maximum difference for the S/SE world-gravity pair is nearly double at $\sim 1700$ feet lower. The maximum differences for E/C and E/PM world-gravity pairs are $\sim 920$ feet and $\sim 800$ feet higher respectively. The magnitude of altitude errors, again, are similar in proportion and direction to the residual tangential accelerations. Since the vehicle is traveling north, the residual gravity component causes the vehicle to slow (sphere) or speed up (ellipsoid). The vehicle reacts by decreasing altitude (sphere) or increasing altitude (ellipsoid) to maintain level flight. The E/SE world-gravity pair remains fairly steady at $\sim 674 \mathrm{ft} / \mathrm{s}$ north velocity. The sphere world-gravity pairs dip to $\sim 664 \mathrm{ft} / \mathrm{s}$ for $\mathrm{S} / \mathrm{C}$ and S/PM and $\sim 656 \mathrm{ft} / \mathrm{s}$ for $\mathrm{S} / \mathrm{SE}$. The E/C and E/PM world-gravity pairs increase speed to $\sim 685 \mathrm{ft} / \mathrm{s}$. The E/PM world-gravity pair breaks away from the $\mathrm{E} / \mathrm{C}$ world-gravity pair toward the higher latitudes because $\mathrm{E} / \mathrm{PM}$ produces a much larger gravitational acceleration near the pole, which is closer to the Earth center than the equator. The same physical effect accounts for the similar but less dramatic decline in altitude shown by the reference world-gravity pair, E/SE.

The error between the non-rotating pairs and the reference world-gravity pair largely is due to the difference in longitude (see Figure 7). This error reflects the other rotation-induced acceleration, the Coriolis acceleration. In this scenario, the Coriolis acceleration begins at zero and increases as the velocity vector of the vehicle turns with the curvature of the Earth. Equation 8 provides a good approximation for the Coriolis acceleration for this scenario. The equation is expressed in the topodetic coordinate system (North-East-Down); the $\mathbf{j}$ unit vector points East.

$$
a_{\text {Coriolis }} \cong 2 \omega U \sin \left(\phi_{g}\right) \mathbf{j}
$$

where $\omega$ is the rotation of the Earth, $U$ is the forward velocity of the vehicle, and $\phi_{\mathrm{g}}$ is the geocentric latitude. The errors in longitude between the rotating and non-rotating world-gravity pairs follow the increasing growth in Coriolis acceleration; the errors remain small until the middle latitudes are reached. The mean Coriolis acceleration for the scenario is $\sim 0.018 \mathrm{~m} / \mathrm{s}^{2}\left(0.059 \mathrm{ft} / \mathrm{s}^{2}\right)$. The mean is the same order of magnitude as the mean residual tangent component of gravity. However, its affect on the vehicle's dynamics is less dramatic. The longitude error of the non-rotating world-gravity pairs is $-2.2^{\circ}$ at the end of the run. This error is much larger than the errors seen in latitude among the rotating world-gravity pairs. However, at the higher latitudes, the distance per degree of longitude is much smaller than the distance per degree of latitude. Thus, the longitude error does not translate into greater position errors for the non-rotating world-gravity pairs versus the rotating world-gravity pairs. Among the rotating world-gravity pairs, the longitude differences are very small, less than $0.36^{\circ}$. The rotating world-gravity pairs show the same groupings and separation as they do in the latitude and altitude charts because, in this scenario, the Coriolis acceleration is largely a function of latitude.

Vehicle dynamics in the aircraft scenario is dominated by differences in tangential accelerations that appear in the Earth-relative reference frames. The errors in the rotating world-gravity pairs are caused by residual tangential components of gravity. The errors in the non-rotating world-gravity pairs are caused by failure to exhibit the Coriolis acceleration. Differences in the normal gravity component are largely inconsequential because they are offset by the aerodynamic lift. Their affect on the simulation is relegated to determining the angle of attack for the vehicle and the initial throttle setting for the engines. Those differences are very small; the range of initial angles of attack, for example, is less than $0.03^{\circ}$.

\section{Conclusions}

The orbital and aircraft scenarios provide contrasting insights into the influence that world-gravity pairs have on the surface relative dynamics of vehicles. The investigation looked at ten world-gravity pairs. The pairs combined the sphere and ellipsoid models of Earth, both with and without rotation modeled, to various gravity or gravitation models. The gravity and gravitation models were constant gravity, free-air reduction gravity, constant gravitation, point-mass gravitation, and gravitation of a symmetric ellipsoid. The rotating ellipsoid with symmetric ellipsoid gravitation (E/SE) was the reference pair against which the other world-gravity pairs were compared. The E/SE pair reproduces the shape and gravity of an Earth in hydrostatic equilibrium as published in WGS84. ${ }^{1,2}$

The orbital scenario was created to examine the surface-relative dynamics of a vehicle when gravity is the only external force. To impart surface-relative behavior, the scenario used a low altitude and initialized all world-gravity pairs using the same surface-relative position, velocity, orientation, and angular velocity. However, the vehicle required an orbital speed to ensure adequate coverage of the Earth surface over a significant period of time. The orbital speed was selected to produce a circular, near polar orbit for the S/PM pair (rotating sphere with point-mass gravitation). The orbital scenario produced a large disparity in surface-relative dynamics among the world-gravity pairs. Only the E/PM pair (rotating ellipsoid with point-mass gravitation) exhibits behavior that could be considered reasonably close to the reference E/SE pair. Simple explanations for the differences require examination in the inertial reference frame. Location errors for the rotating world-gravity pairs are dominated by differences in orbital period and orbit shape. Location errors for the non-rotating world gravity pairs are caused by the lack of surface 
rotation below an orbit fixed in inertial space. The differences among the world-gravity pairs only highlights that a zero-drag vehicle traveling an orbital velocity is not bound to the Earth. The resulting simulation does not exhibit the characteristics of a surface-interacting simulation. The scenario provides little additional guidance on using the world-gravity pairs. The scenario only reinforces current practice of world and gravitation model selection for orbital vehicles, i.e. model rotation and do not use constant gravitation. The scenario does provide practical evidence that coupling geopotential models, like the symmetric ellipsoid gravitation, with the rotating sphere degrades, not improves performance, relative to the lower-fidelity point-mass gravitation model.

The aircraft scenario does produce results that can only be explained in the Earth-fixed frame and, therefore, retains the characteristics of a surface interacting simulation. Location errors in the rotating worlds are primarily the result of residual surface-tangent components of gravity. This residual tangential gravity appears when the gravitation model and the surface model don't create tangent components of centrifugal acceleration and tangent components of gravitation that negate each other. This balance is only achieved in the reference pair, E/SE. A lower fidelity surface or a lower fidelity gravitation model disrupts this balance. The non-rotating worlds do not experience these tangential accelerations because they produce no centrifugal acceleration and the gravity models produce accelerations that are always normal to the surface. The non-rotating worlds, however, also do not generate Coriolis acceleration. The missing Coriolis acceleration accounts for their location errors. For this direct flight from the equator to the North pole, the residual tangential gravity appears to have a greater influence on surface-relative dynamics than the missing Coriolis acceleration. As a lower fidelity alternative to E/SE, the non-rotating worlds actually provide better performance than the rotating worlds. The outcome does not match the expected order of gravity fidelity presented in section II.C. In fact, the order is first driven by world model (ENR $\rightarrow \mathrm{SNR} \rightarrow \mathrm{E} \rightarrow \mathrm{S}$ ) and the gravity or gravitation model is largely inconsequential. The one exception is the S/SE pair, which again, demonstrates worse performance than the other rotating sphere pairs; thus it should be avoided. The E/PM pair, which analytically produces poor fidelity as evidenced in Figure 9, performs almost equally to the E/C pair. This scenario does not support a recommendation to avoid this pair.

The aircraft scenario results results may not hold for other trajectories, particularly trajectories that cross from East to West which experience Coriolis accelerations throughout the trajectory. But, the explanations give clues to the expected behavior in other trajectories. The rotating world pairs would appear to have an advantage with EastWest trajectories in the lower and upper latitudes where the residual tangential acceleration is small. But, residual tangential gravity is highest in the mid-latitudes and may still have a larger effect than failure to exhibit Coriolis acceleration. The tangential gravity is a function only of location. The Coriolis acceleration is primarily a function of vehicle velocity. Therefore, vehicle velocity may determine whether the rotating or non-rotating worlds produce less error in trajectories covering the mid-latitudes. Results may also change at higher altitude where differences among the gravity and gravitation models are larger. Testing these hypotheses with simulation data is left to future work. Future work may also examine the errors of the world-gravity pairs in launch and entry scenarios or scenarios that include the full range of flight from takeoff to landing. 


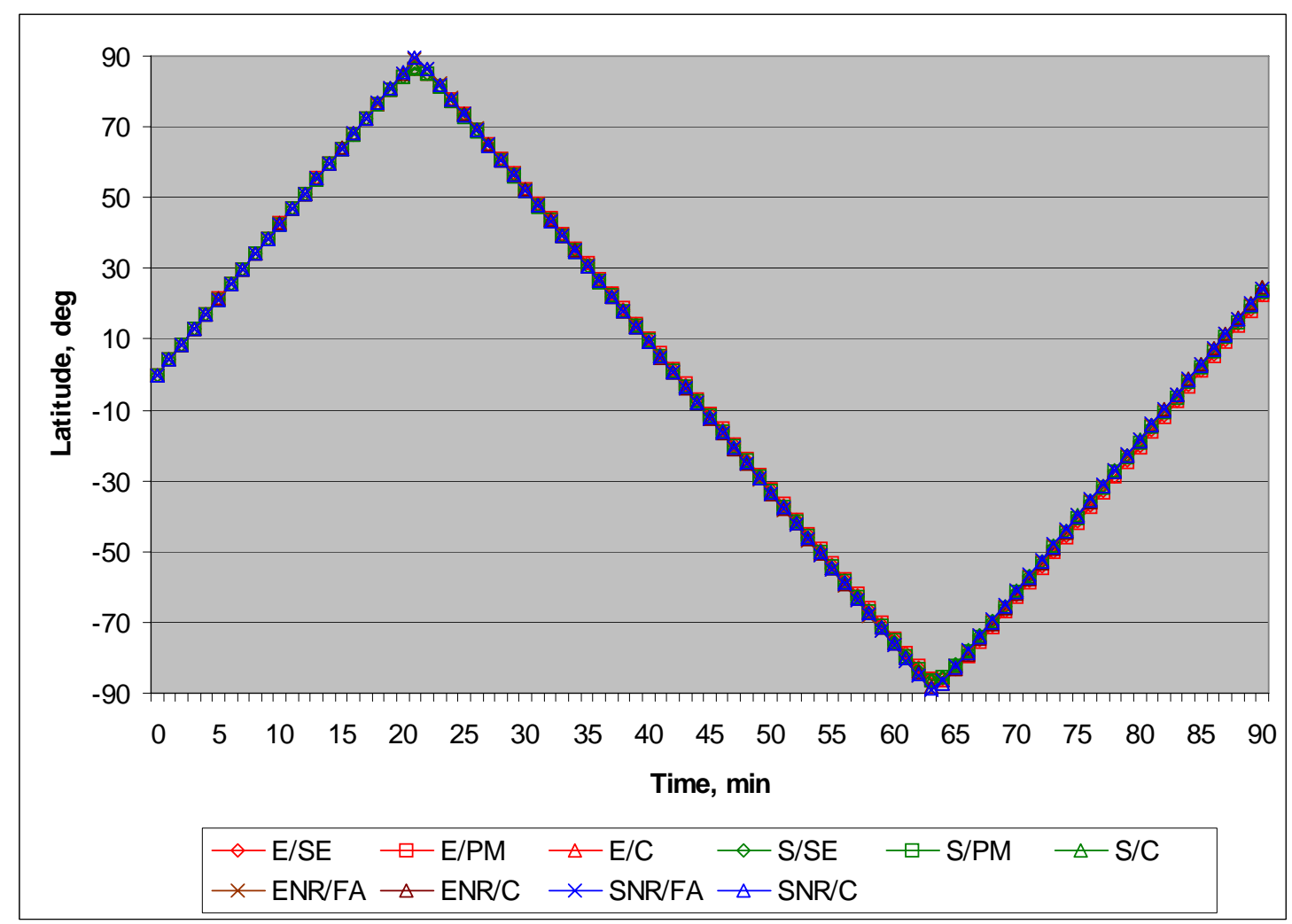

Figure 1 Orbital Secnario - Latitude vs. Time

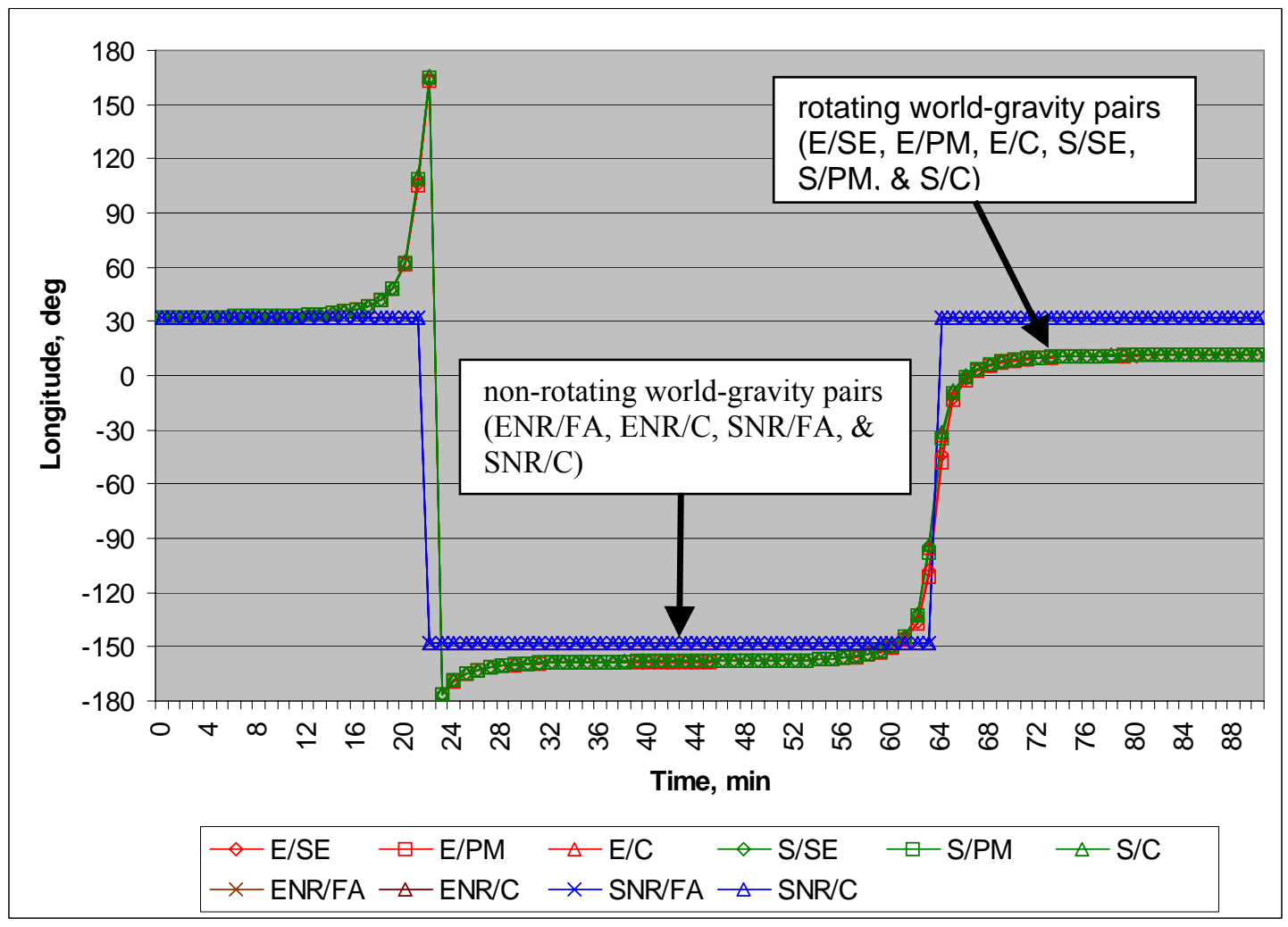

Figure 2 Orbital Scenario - Longitude vs. Time 


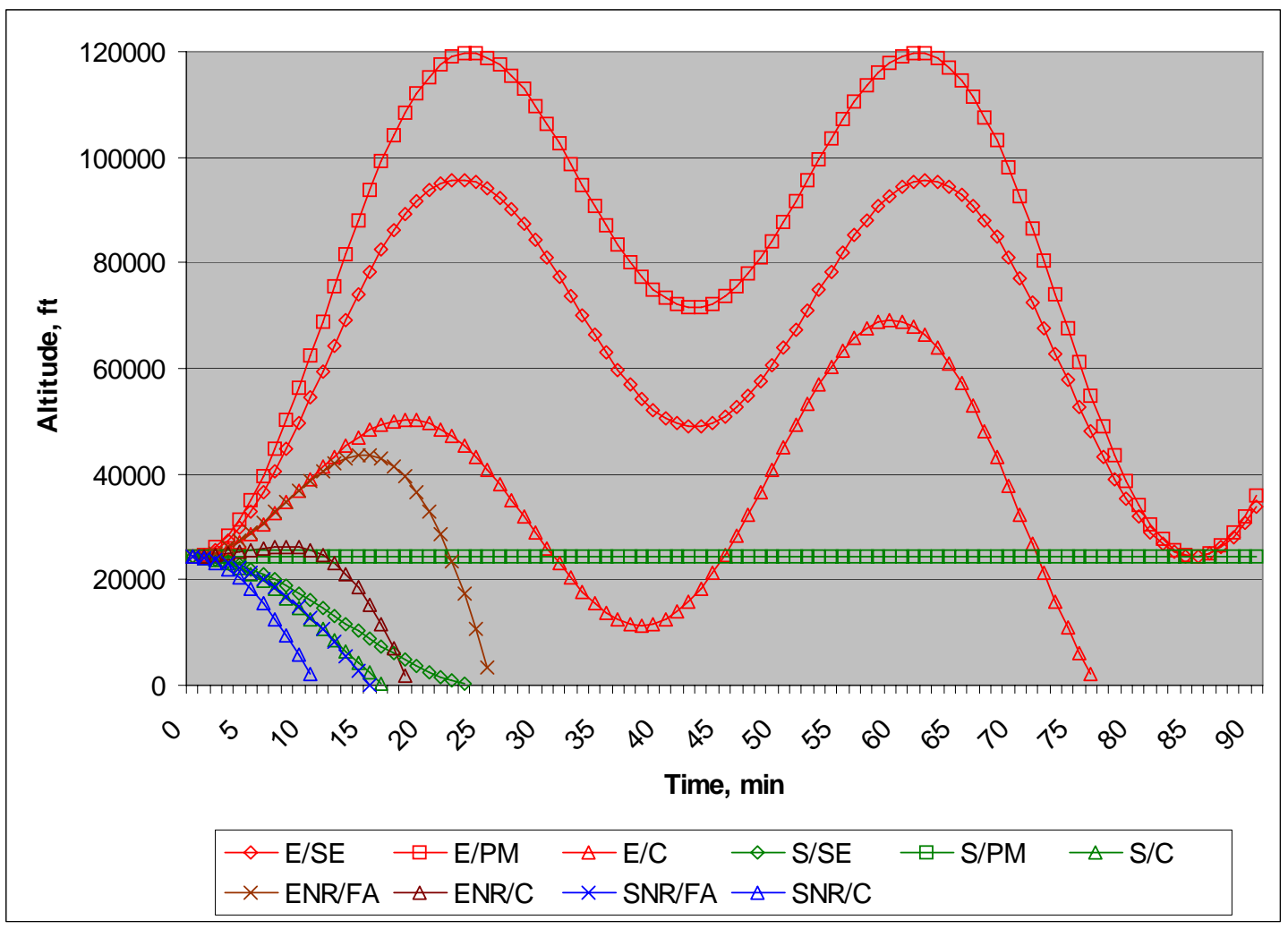

Figure 3 Orbital Scenario - Altitude vs. Time

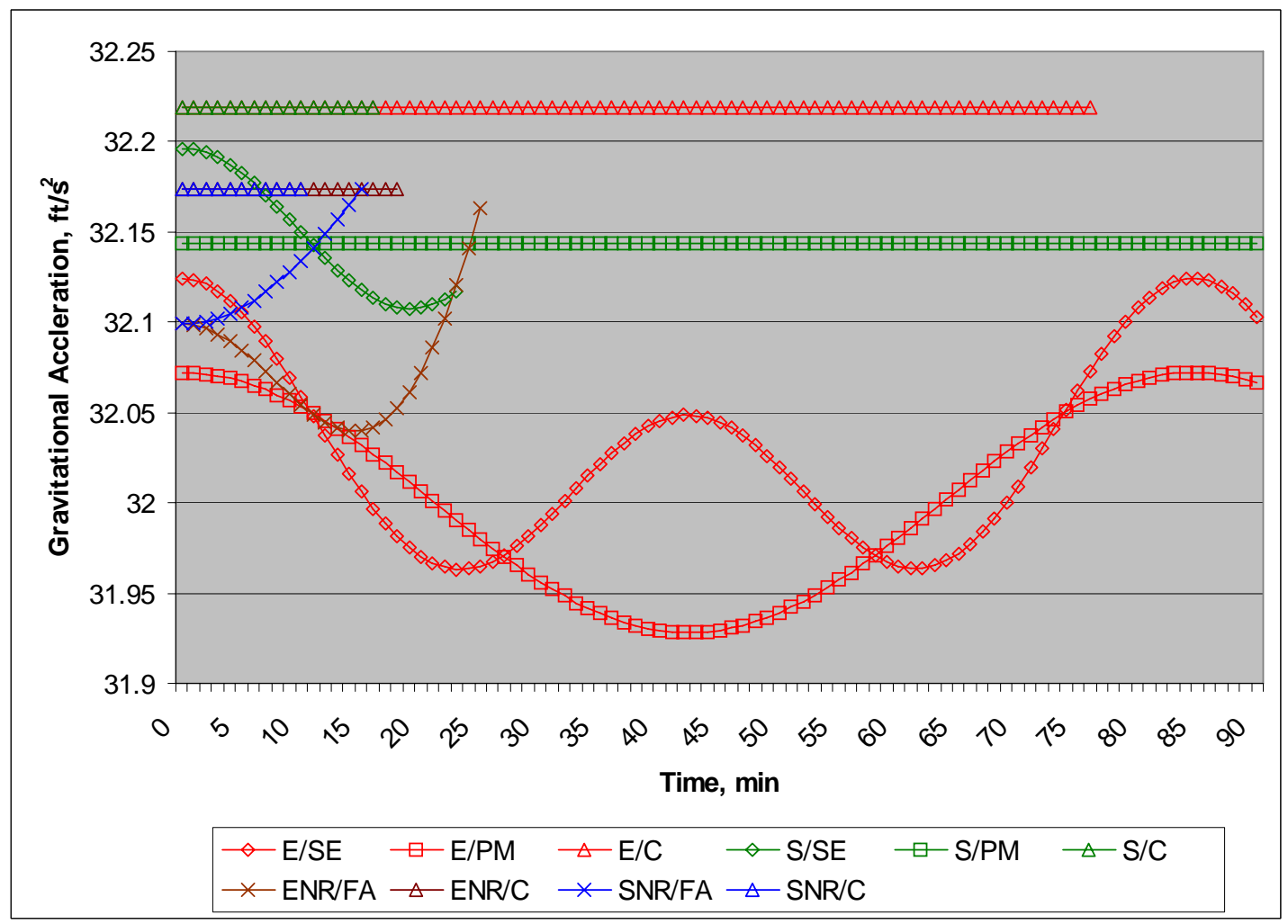

Figure 4 Oribtal Scnenario - Magnitude of "Inertial" Gravitational Acceleration vs. Time 


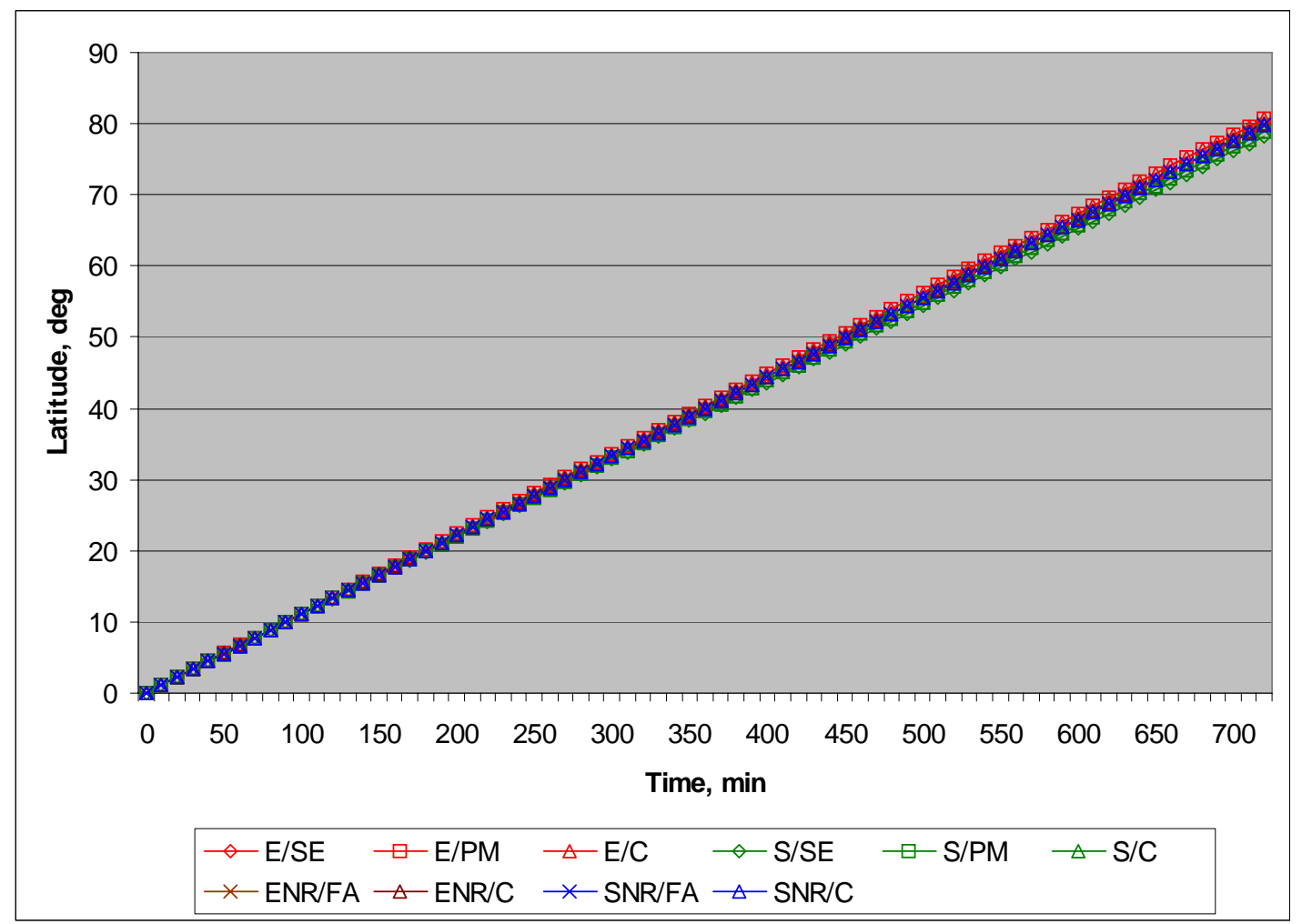

Figure 5 Aircraft Scenario - Latitude vs. Time

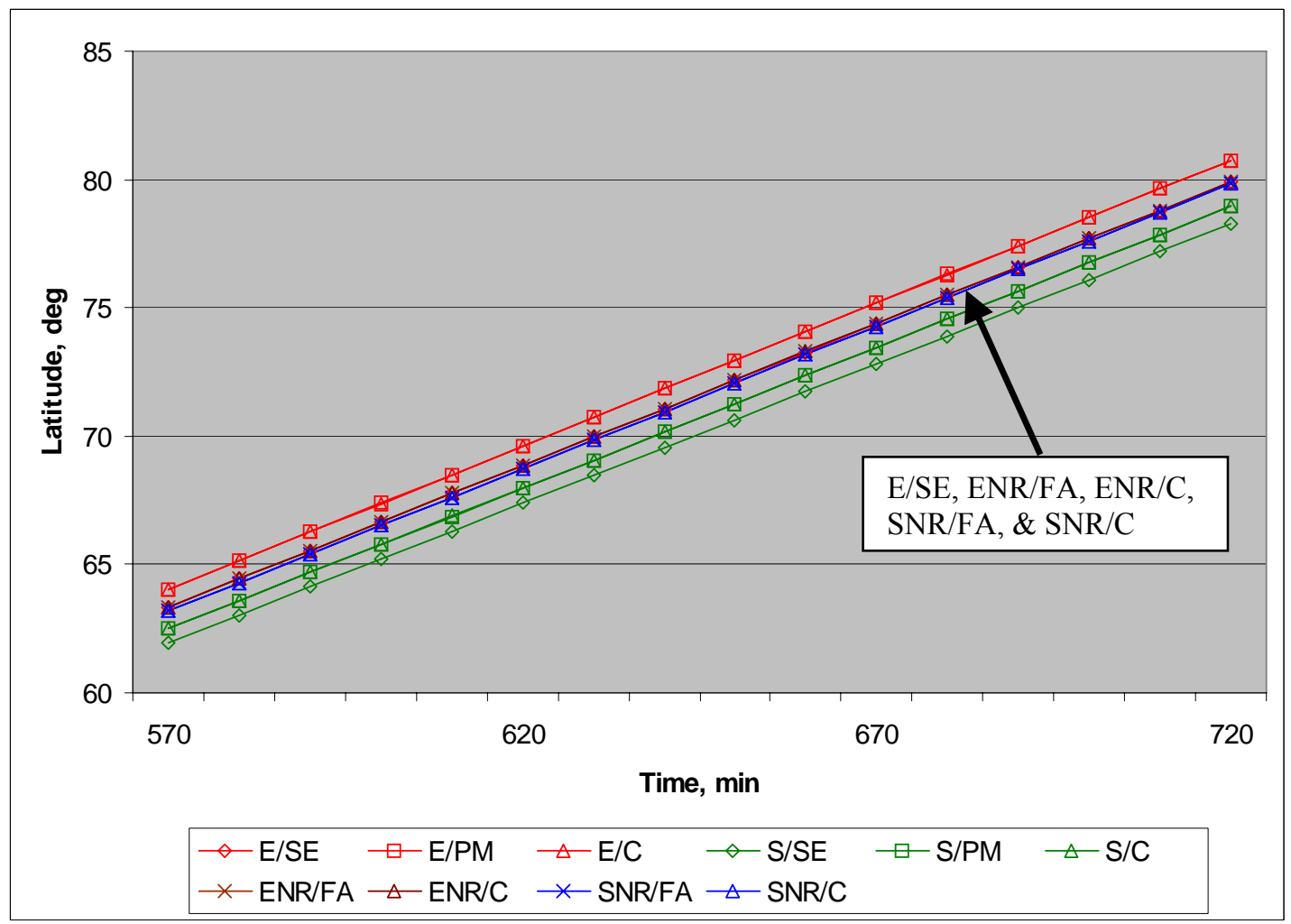

Figure 6 Aircraft Scenario - Latitude vs. Time (Last 150 minutes) 


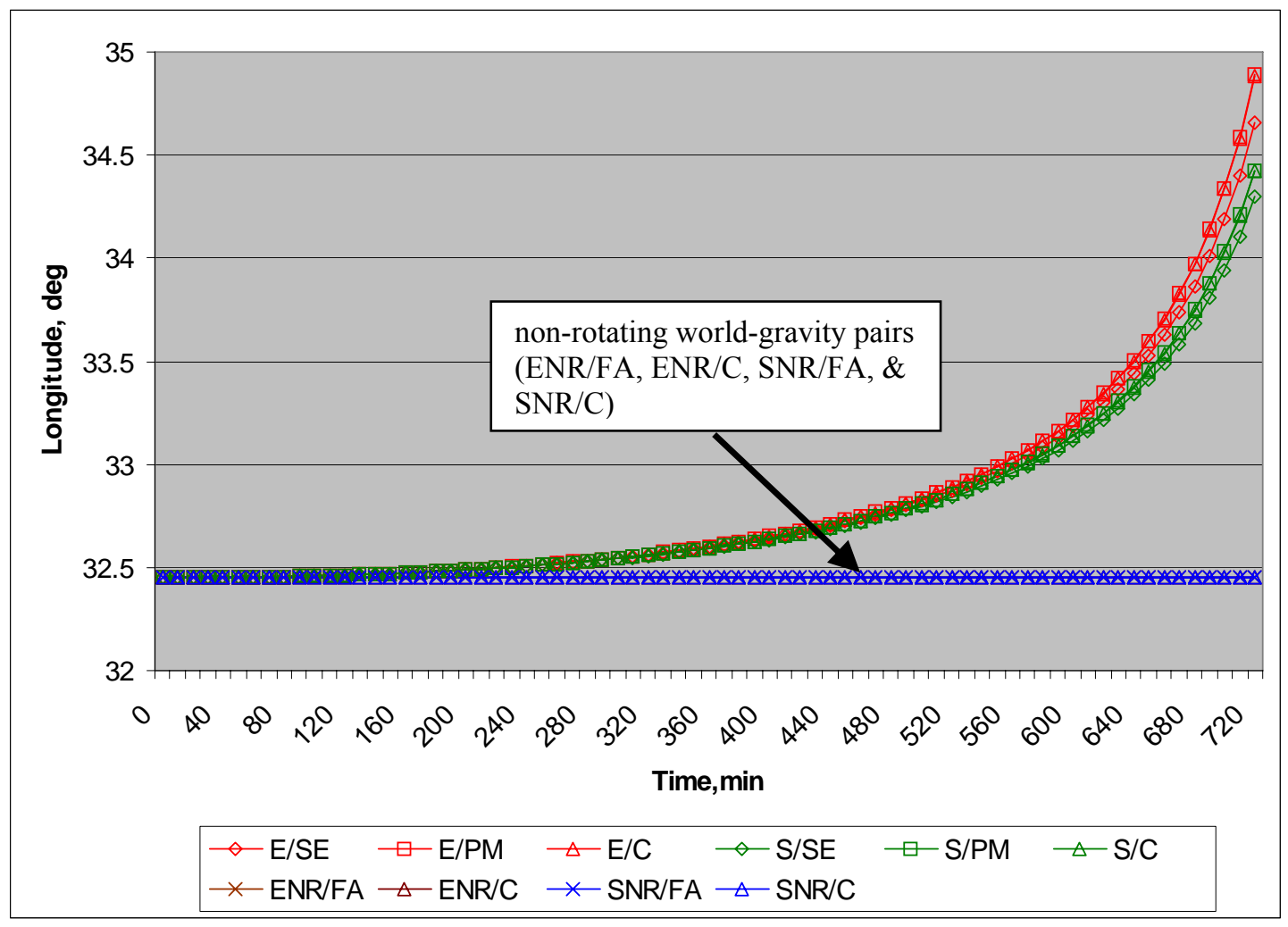

Figure 7 Aircraft Scenario - Longitude vs. Time

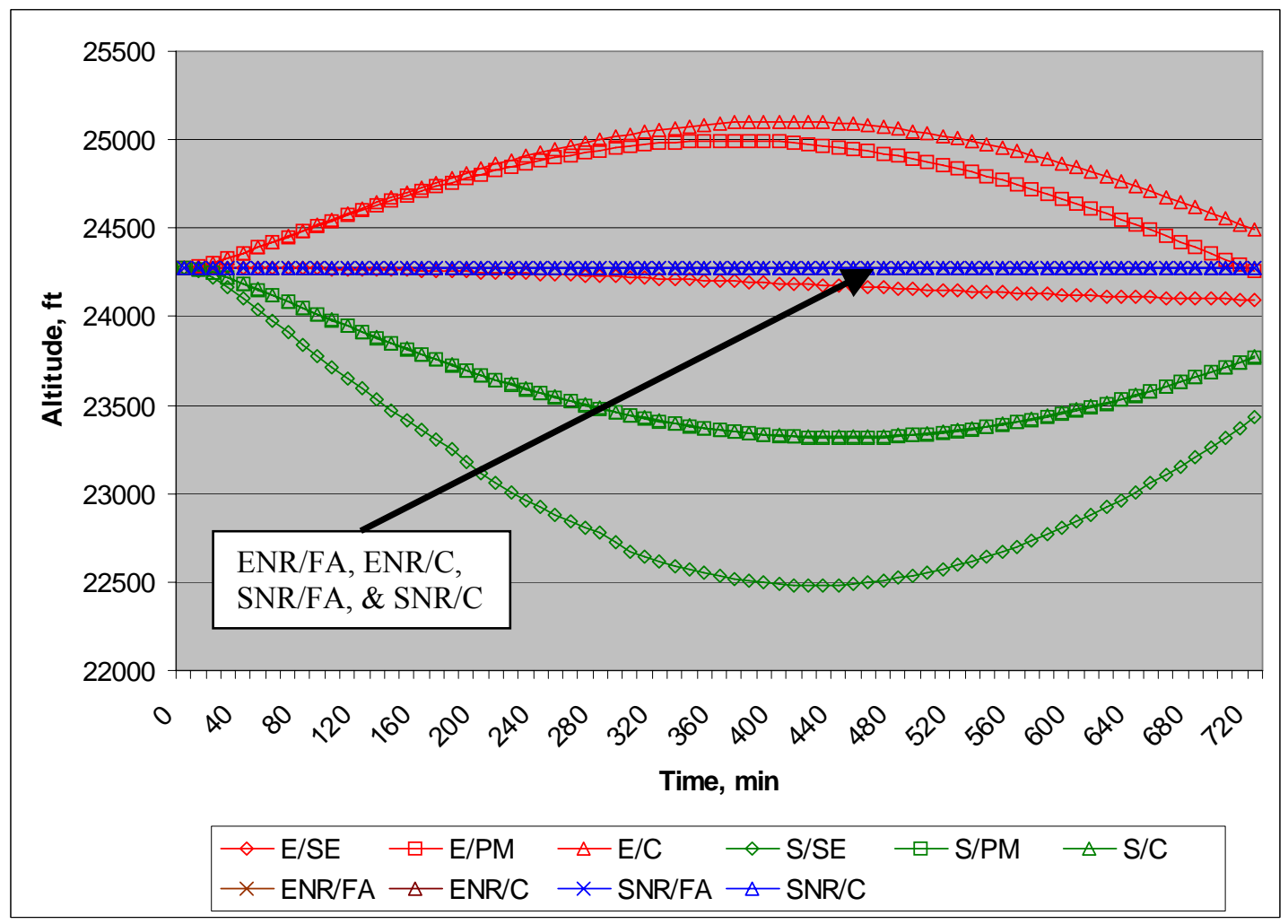

Figure 8 Aircraft Scenario - Altitude vs. Time 


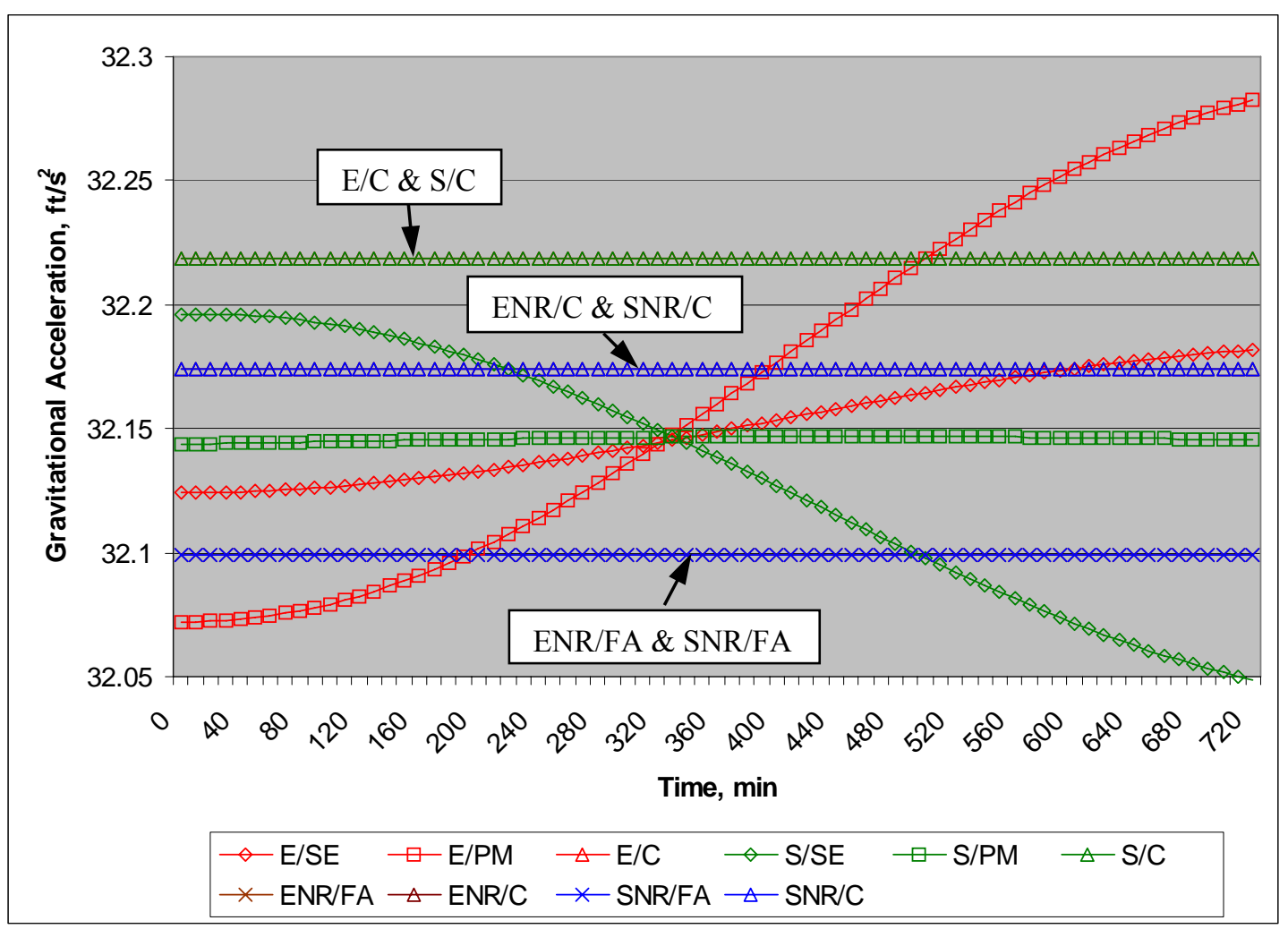

Figure 9 Aircraft Scenario - Magnitude of Inertial Gravitational Acceleration

\section{References}

1 Madden, Michael M. "Gravity Modeling for Variable Fidelity Environments”, AIAA-2006-6730, AIAA Modeling and Simulation Technologies Conference, Keystone, CO, August 21-24, 2006.

2 "Department of Defense World Geodetic System 1984", National Imagery and Mapping Agency, NIMA TR8350.2, Third Edition, Amendment 1, 3 January 2000.

3 Allen, William H. (Ed.), "Dictionary of Technical Terms for Aerospace Use", National Aeronautics and Space Administration, Washington D.C., NASA SP-7, First Edition, 1965. Also available as an online database at URL: http://roland.lerc.nasa.gov/ dglover/dictionary/content.html [cited July 19, 2006].

4 Hofmann-Wellenhof, Bernhard and Moritz, Helmut, Physical Geodesy, SpringerWeinNewYork, New York, 2005, ISBN 3-211-23584-1. Sections Preface, 1.7, 2.1, 2.10.

5 Moritz, H. “Geodetic Reference System 1980”, Journal of Geodesy, Vol. 74, No.1, 2000, pp. 128-133.

6 R. Leslie, D. Geyer, K. Cunningham, M. Madden, P. Kenney, and P. Glaab, "LaSRS++: An Object-Oriented Framework for Real-Time Simulation of Aircraft," AIAA Modeling and Simulation Technologies Conference and Exhibit, August 1998, Boston, Massachusetts, AIAA-98-4529. 